\title{
$\mathrm{Q} /$ Does cervical membrane stripping in women with group B Streptococcus put the fetus at risk?
}

\section{EVIDENCE-BASED ANSWER}

A/ NO DIRECT EVIDENCE points to fetal harm from cervical membrane stripping (CMS) to induce labor in term pregnancies complicated by group B Strep- tococcus (GBS) colonization (strength of recommendation [SOR]: B, a Cochrane systematic review).
Mark S. Crago, MD, PhD Robert Gauer, MD

Residency Faculty, Womack Army Medical Center, Department of Family Medicine, Ft. Bragg, NC

\section{Jori Frazier, MLIS}

Carolinas Healthcare System, Charlotte, NC

ASSISTANT EDITOR Richard Guthmann, MD, MPH

University of Illinois at Chicago, Advocate Illinois Masonic Family Medicine Residency

\section{Evidence summary}

A Cochrane review of 22 trials ( $\mathrm{N}=2797$ ) comparing CMS with no CMS in uncomplicated term deliveries demonstrated no significant differences in fetal outcomes. ${ }^{1}$ The groups showed similar rates of maternal infection and fever (relative risk $[\mathrm{RR}]=1.05$; 95\% confidence interval $[\mathrm{CI}], 0.68-1.65)$, neonatal infection (RR $=0.92 ; 95 \% \mathrm{CI}, 0.30-2.82$ ), and Apgar scores $<7$ at 5 minutes ( $\mathrm{RR}=1.13$; 95\% CI, 0.53-2.43). Two perinatal deaths were reported in each group. The review was limited by relatively small trials and heterogeneity between trial results, suggesting the possibility of publication bias.

Most of the studies included in the metaanalysis didn't specifically include or exclude women with GBS colonization, nor did the review subanalyze patients into a GBS-positive and GBS-negative arm. Considering that GBS colonization was reported in $19 \%$ to $26 \%$ of pregnancies, it's likely that GBS colonization was present in both CMS and control groups in the review. ${ }^{2,3}$

\section{Study shows no CMS effects, but may be underpowered}

A randomized prospective study ( $\mathrm{N}=98)$ included in the Cochrane review specifically considered the effects of CMS and maternal GBS colonization. ${ }^{4}$ Colonization rates for the study were $17 \%$ (9/44 in the study group, 8/54 in the control group). Women in the study group underwent weekly CMS beginning at 38 weeks of gestation; the control group didn't undergo CMS. Repeat GBS testing was performed at 40 weeks for all patients with initial GBS-negative cultures.

Three patients were GBS-positive on repeat testing (one in the study group, 2 in the control group). No admissions to the neonatal intensive care unit or neonatal infections occurred in either group. The study may have been underpowered to detect any effect, however. ${ }^{4}$

\section{Recommendations}

The American College of Obstetricians and Gynecologists' 2009 Practice Bulletin on induction of labor states that the data are insufficient to either recommend or discourage CMS to induce labor in women who are GBS-positive. ${ }^{5}$

The 2009 Department of Veterans Affairs/Department of Defense Clinical Practice Guideline for Pregnancy Management also cites insufficient data to support or oppose CMS in GBS-positive term pregnant women. ${ }^{6}$ 


\section{References}

1. Boulvain M, Stan CM, Irion O. Membrane sweeping for induction of labor. Cochrane Database Syst Rev. 2005;(1):CD000451.

2. Regan JA, Klebanoff MA, Nugent RP. The epidemiology of group B streptococcal colonization in pregnancy. Vaginal Infections and Prematurity Study Group. Obstet Gynecol. 1991;77:604-610.

3. Yancey MK, Schuchat A, Brown LK, et al. The accuracy of late antenatal screening cultures in predicting genital group B streptococcal colonization at delivery. Obstet Gynecol. 1996;88:811-815.

4. Netta D, Visintainer P, Bayliss P. Does cervical membrane stripping increase maternal colonization of group B streptococcus? Am J Obstet Gynecol. 2002;187:S221. [Abstract.]

5. American College of Obstetricians and Gynecologists Committee on Practice Bulletins-Obstetrics. ACOG Practice Bulletin. Clinical management guidelines for obstetrician-gynecologists. Induction of labor. Obstet Gynecol. 2009;114:386-397.

6. United States Department of Veterans Affairs and US Department of Defense, Pregnancy Management Working Group. VA/DoD clinical practice guideline for pregnancy management, 2009. Available at: www.healthquality.va.gov/up/mpg v2_1_full.pdf. Accessed April 16, 2010. 